\title{
Performance of the CMS electromagnetic calorimeter during the LHC Run II and its role in precision physics measurements
}

\author{
Marco Cipriani* on behalf of the CMS Collaboration \\ Sapienza Università di Roma \& INFN Romal \\ E-mail: marco.cipriani@cern.ch
}

\begin{abstract}
Many physics analyses using the Compact Muon Solenoid (CMS) detector at the LHC require accurate, high resolution electron and photon energy measurements. Particularly important are the decays of the Higgs boson resulting in electromagnetic particles in the final state, as well as the searches for very high mass resonances decaying into energetic photons or electrons. Following the excellent performance achieved in Run I at center-of-mass energies of 7 and $8 \mathrm{TeV}$, the CMS electromagnetic calorimeter (ECAL) is operating at the LHC with proton-proton collisions at 13 $\mathrm{TeV}$ center-of-mass energy. The instantaneous luminosity delivered by the LHC during Run II has achieved unprecedented values, using $25 \mathrm{~ns}$ bunch spacing. High pileup levels necessitate a retuning of the ECAL readout and trigger thresholds and reconstruction algorithms, to maintain the best possible performance in these more challenging conditions. The energy response of the detector must be precisely calibrated and monitored to achieve and maintain the excellent performance obtained in Run I in terms of energy scale and resolution. A dedicated calibration of each detector channel is performed with physics events exploiting electrons from $\mathrm{W}$ and $\mathrm{Z}$ boson decays, photons from $\pi^{0} / \eta$ decays, and from the azimuthally symmetric energy distribution of minimum bias events. This contribution describes the calibration strategies and the performance of the CMS ECAL throughout Run II and its role in precision physics measurements with CMS involving electrons and photons.
\end{abstract}

Sixth Annual Conference on Large Hadron Collider Physics (LHCP2018)

4-9 June 2018

Bologna, Italy

${ }^{*}$ Speaker. 


\section{Introduction}

The Compact Muon Solenoid (CMS) [1] at the CERN Large Hadron Collider (LHC) is a general purpose detector designed to discover the Standard Model (SM) Higgs boson and search for new physics beyond the SM. The excellent performance in the reconstruction and identification of high energy photons and electrons has played a key role in the observation of the Higgs boson and the study of its properties [2] during the LHC Run I. CMS features a high-resolution, homogeneous electromagnetic calorimeter (ECAL) [3] comprising 61200 crystals of lead tungstate $\left(\mathrm{PbWO}_{4}\right)$ in the central barrel detector (EB) complemented by 7324 crystals in each of the two endcaps (EE). Thanks to the properties of $\mathrm{PbWO}_{4}$ (Molière radius of $2.19 \mathrm{~cm}$ and a radiation length $X_{0}=$ $0.85 \mathrm{~cm}$ ), ECAL is characterized by high granularity and excellent longitudinal containment of the electromagnetic shower of electrons and photons with energies up to the $\mathrm{TeV}$ scale. In addition, the decay time of the scintillation light is about $25 \mathrm{~ns}$, which guarantees a fast detector response.

The barrel covers the region within $|\eta|=1.48$, while the endcaps extend the coverage up to $|\eta|<3.0$. A preshower detector (ES), based on lead absorbers equipped with silicon strip sensors, is placed in front of EE. It covers the region $1.65<|\eta|<2.6$ and helps resolve the signals of high-energy photons from the decays of neutral pions into two close photons, improving also the measurement of the position of the electromagnetic deposit in EE. ECAL is installed inside the CMS superconducting solenoid, which provides a magnetic field of 3.8 T. The CMS silicon tracker, located inside ECAL, detects charged particles up to $|\eta|=2.5$ and allows the identification of electrons and photons.

Maintaining and possibly improving the excellent ECAL performance during Run I in the harsher environment of the LHC Run II and beyond is vital for all the physics analyses that include photons or electrons in their final state. Achieving this goal requires a continuous effort in the operation, monitoring and calibration, and simulation of the calorimeter. EB and EE will keep operating without major changes during Run III as well. After that phase, EB will be upgraded with new electronics, while EE will be completely replaced by a new detector.

\section{Electron and photon reconstruction}

Electrons and photons deposit their energy in several ECAL crystals and are reconstructed through a clustering algorithm. The magnetic field bends the trajectory of electrons along the $\phi$ angle direction. Therefore, basic clusters are extended along $\phi$ to form superclusters (SC) and recover additional energy deposits produced by electron bremsstrahlung or photon conversions in the tracker. The SC energy is estimated as $E_{e, \gamma}=F_{e, \gamma} \cdot G \cdot \sum_{i}\left(S_{i}(t) \cdot C_{i} \cdot A_{i}\right)$, where $F_{e, \gamma}$ includes corrections to the clustered energy and $G$ is an $\mathrm{ADC}$ to $\mathrm{GeV}$ conversion factor. The sum runs over the channels in the cluster: $A_{i}$ is the amplitude measured in the $i$-th channel; $S_{i}(t)$ is a timedependent correction for variations of channel response due to changes in crystal transparency; $C_{i}$ is a relative calibration constant accounting for differences in the light yield and photodetector response of each channel.

The output of the photodetectors is digitized by a 12 bit ADC running at $40 \mathrm{MHz}$ and the signal amplitude is reconstructed from a series of 10 samples, one every $25 \mathrm{~ns}$. The observed pulse shape is distorted by the energy deposited by particles originating from simultaneous proton collisions 
(pileup) and the estimated signal amplitude is then biased. Moreover, the time spacing between colliding proton bunches was reduced from 50 to $25 \mathrm{~ns}$ during Run II, enhancing the contribution of out-of-time pileup (OOT). To mitigate the impact of OOT on the measured amplitude, an improved algorithm, named multifit, was developed during Run II [4]. The multifit models the observed pulse shape as the sum of one in-time and up to 9 out-of-time pulse amplitudes. The in-time signal amplitude is then extracted through the minimization of a $\chi^{2}$ variable. An example of a fit in EE is shown in Fig. 1a. The total pulse, overlaid with the observed signal, is obtained as the sum of the fitted pulses. The in-time pulse peaks at the sixth time sample corresponding to the in-time bunch crossing. The other out-of-time pulses peak at different time samples and represent the energy contribution from OOT. All the pulse templates have the same shape and only differ by a $25 \mathrm{~ns}$ shift on the horizontal axis.

The energy of an SC is corrected using a multivariate analysis technique (MVA) trained on simulations, taking, among other variables, the coordinates of the crystals and the shower shapes as input. The MVA addresses the energy containment inside the SC and corrects for several effects, such as the energy loss due to interactions with material in front of ECAL, energy leakage in gaps between crystals and spurious energy deposits due to in-time pileup events. The output correction factor $F_{e, \gamma}$ is tuned separately on electrons and photons to account for differences due to photon conversions in the material upstream of ECAL or electron bremsstrahlung. Finally, the absolute energy scale $G$ is set to match the invariant mass from simulated $Z \rightarrow e e$ events.

\section{Crystals response monitoring and inter-calibration}

ECAL crystals undergo a change of transparency due to radiation damage during periods of LHC operation. This effect induces a change in the energy response of each channel, which is constantly monitored and corrected for by a dedicated laser system. Figure $1 \mathrm{~b}$ shows the relative response of ECAL crystals to laser light as a function of time, for different regions of $\eta$. The loss of transparency is larger at higher $\eta$ due to heavier radiation damage, and is partially recovered during periods with no collisions. The stability of the energy scale is monitored using the diphoton invariant mass distribution in events with $\pi^{0} \rightarrow \gamma \gamma$ decays. Electrons from decays of $\mathrm{Z}$ and $\mathrm{W}$ bosons are used as well, exploiting the $Z \rightarrow e e$ invariant mass or the ratio of the electron energy measured with ECAL and its momentum measured with the tracker. The time-dependent drift in these observables is removed after applying the laser monitoring correction, thus validating the goodness of such correction. Each point in the $\pi^{0}$ monitoring history is obtained from a fit to about $5 \times 10^{5} \pi^{0}$ 's collected every 5 minutes of data-taking by a special data stream. $\mathrm{Z}$ and $\mathrm{W}$ bosons are selected with much lower rate, but generate electrons whose energy is closer to physics events. The energy scale during 2017 was stable within $0.1 \%$ (0.2\%) in EB (EE) [6].

The energy resolution of a calorimeter for high energy electron and photon showers is dominated by the constant term. The main contribution comes from the accuracy of the inter-calibration (IC) constants. The IC procedure aims at equalizing the variations of the measured energy among different ECAL channels. Several methods based on physics processes are used to provide an IC constant for each channel [5]: $\pi^{0} \rightarrow \gamma \gamma, Z \rightarrow e e, E / p$. Each method compares a given observable, measured using only ECAL information, with a physics reference. The azimuthal symmetry in the 


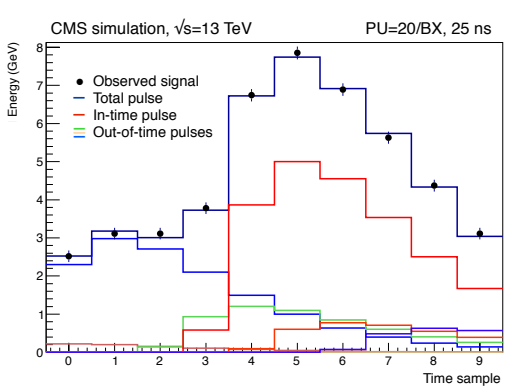

(a) multi-fit in EE

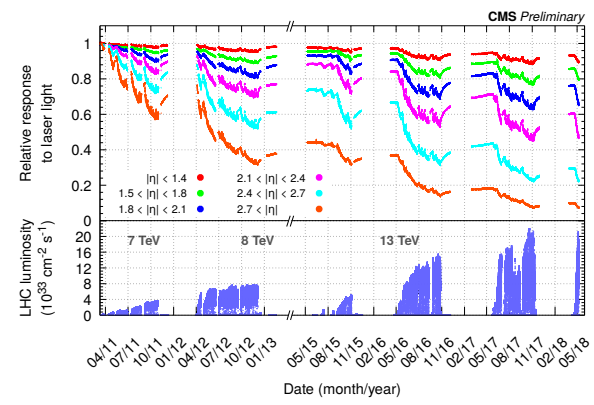

(b) Relative response to laser light

Figure 1: (a) fitted pulse shapes in EE for simulated events. (b) ECAL crystals relative response to laser light as a function of time, for different regions of pseudorapidity. The lower panel shows the instantaneous luminosity reached by LHC during operation. Taken from [4, 6].

energy distribution of minimum bias events (known as $\phi$-symmetry) is used as well, but provides lower precision compared to other methods.

The calibration with $\pi^{0}$ 's exploits the position of the peak in the invariant mass distribution of unconverted photon pairs. Events used for this calibration are selected by a dedicated trigger stream which saves only limited information in the vicinity of the selected photon candidates. This feature minimizes the usage of the CMS readout bandwidth and storage space, allowing the stream to run with a high rate of several $\mathrm{kHz}$ in $\mathrm{EB}$ and $\mathrm{EE}$. The $E / p$ method uses prompt electrons from decays of $\mathrm{W}$ and $\mathrm{Z}$ bosons and is based on the comparison of the electron energy measured with ECAL $(E)$ and the electron momentum measured with the tracker $(p)$. The $Z \rightarrow e e$ method is similar to the $\pi^{0}$ one, but relies on the invariant mass of the $\mathrm{Z}$ resonance. Events used for the calibration with $\mathrm{W}$ and $\mathrm{Z}$ bosons are collected using standard CMS triggers with a rate of some tens of $\mathrm{kHz}$.

IC constants were derived using data collected in 2017. The precision reached by each calibration method in EB is shown in Fig. 2a. The precision obtained in the combination is better than $0.3 \%$ for $|\eta|<0.8$ and is lower than $1 \%$ in the rest of EB. The uncertainty on the IC constants provided by each method is generally dominated by systematic uncertainties, except at high $\eta$. The $\pi^{0}$ method reaches the lowest statistical uncertainty, ranging between $0.1 \%$ and $0.5 \%$ in EB (larger at higher $\eta$ ) and about $1 \%$ in EE using less than $10 \mathrm{fb}^{-1}$ of data, allowing to derive a set of IC potentially every month of data-taking. However, it is limited by larger systematic uncertainties due to energy containment and pileup with respect to other methods using $\mathrm{W}$ and $\mathrm{Z}$ bosons. An example of reconstructed $\pi^{0}$ mass distribution in central EB is shown in Fig. $2 b$.

The calibration based on 2017 data helped improve the electron energy resolution with respect to the calibration conditions available at the end of 2017. Figure $2 \mathrm{c}$ shows the energy resolution for low bremsstrahlung electrons, as a function of electron pseudorapidity. The observed resolution gets worse at higher $\eta$ in EB due to the larger amount of material upstream of ECAL, although a general improvement bigger than $10 \%$ is observed in the entire acceptance region.

\section{Conclusions}

ECAL has shown excellent performance in the reconstruction of electrons and photons during Run II thanks to a continuous effort in the operation, monitoring and calibration of the detector. 


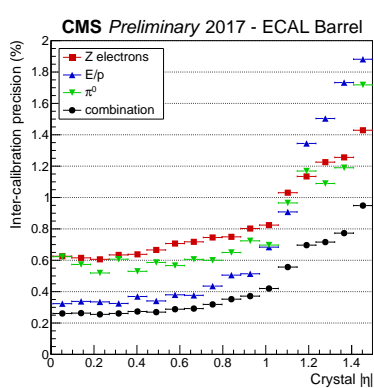

(a) IC constants precision

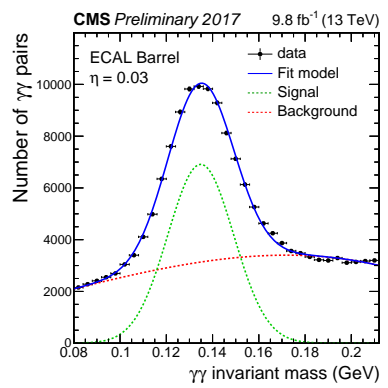

(b) $\pi^{0}$ mass in EB

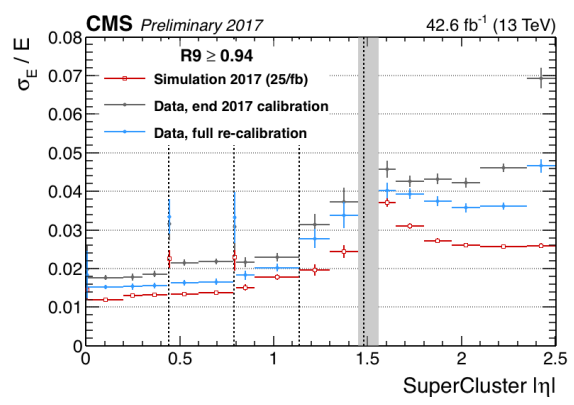

(c) Electron energy resolution

Figure 2: (a) precision of the inter-calibration (IC) constants and their combination in EB. (b) example of a $\pi^{0}$ mass peak in one EB channel. Data points are fitted with the sum (solid line) of a signal and background components (dashed lines) modeled as a Gaussian function and a Chebychev polynomial respectively. (c) energy resolution measured from the invariant mass of electron pairs in $Z \rightarrow e e$ events for low bremsstrahlung electrons $\left(R_{9}>0.94\right.$, where $R_{9}$ is defined as the ratio of the energy measured in a $3 \times 3$ matrix of crystals centred on the SC crystal with the highest energy and the total SC energy [5]). The resolution is significantly improved after applying the calibration constants derived using 2017 data. Taken from [6]

The calibration campaign made at the end of 2017 has improved the energy resolution by more than $10 \%$ on average with respect to the previous available calibration.

The excellent performance of ECAL is paramount for the Higgs boson physics program. Indeed, electron and photons provide a clean experimental signature and allow the measurement of the properties of the Higgs boson with unprecedented precision. Moreover, they are fundamental ingredients to probe the possible existence of new heavy resonances decaying into lepton or photon pairs, due to much better $e / \gamma$ resolution with respect to muons for energies of the order of the TeV. In addition, ECAL will also contribute to many standard model precision measurements at lower energy, such as the $\mathrm{W}$ boson mass, complementing the muon channels.

\section{References}

[1] S. Chatrchyan et al. [CMS Collaboration], The CMS experiment at the CERN LHC, JINST 3, S08004 (2008).

[2] V. Khachatryan et al. [CMS Collaboration], Observation of the diphoton decay of the Higgs boson and measurement of its properties, Eur. Phys. J. C 74, (2014) 3076 [arXiv:1407.0558v2 [hep-ex]].

[3] [CMS Collaboration], CMS: The electromagnetic calorimeter. Technical design report, CERN-LHCC-97-33, CMS-TDR-4.

[4] E. Di Marco [CMS Collaboration], CMS electromagnetic calorimeter calibration and timing performance during LHC Run I and future prospects, CMS-CR-2014-410 (2014); http://cds.cern.ch/record/1975982

[5] S. Chatrchyan et al. [CMS Collaboration], Energy calibration and resolution of the CMS electromagnetic calorimeter in pp collisions at $\sqrt{s}=7$ TeV, JINST 8, P09009 (2013) [arXiv:1306.2016v2 [hep-ex]].

[6] CMS Collaboration, CMS ECAL with 2017 data, CMS-DP-2018-015 (2018); https://cds.cern.ch/record/2319285 